\title{
DEVELOPMENTAL COXA VARA
}

\author{
A. B. Le Mesurier, Toronto, Canada *
}

Excellent papers have appeared from time to time on the subject of developmental coxa vara, sometimes under the titles of congenital, infantile, or cervical coxa vara. Despite this fairly extensive literature, and probably because the condition is not common, its real nature and severity have seldom been recognised as soon as they should be, and treatment has not usually been started until there was marked deformity. The condition is characterised by the development, at the age of about three or four years, of a limp or waddle which is usually painless and is progressive. A varying degree of coxa vara deformity is present on one or both sides, but the most striking feature is the radiographic appearance of a gap in the neck of the femur just distal to the epiphyseal line, together with a bend in the femoral neck at this level. This X-ray appearance is, in itself, almost sufficient to establish the diagnosis as soon as the patient is first seen.

Frequency-The condition is rare, and few large series of cases have been reported. Statistics of the Hospital for Sick Children, Toronto, show that in twelve years, 1936 to 1947 inclusive, fifteen patients with developmental coxa vara were admitted. In the same period 197 new patients were admitted with congenital dislocation of the hip joint. Thus we have seen one case of coxa vara for every thirteen cases of congenital dislocation. It is to be noted, however, that congenital dislocation of the hip joint does sometimes run in families and that it is more common in some districts than others. Moreover, developmental coxa vara mav also run in families. It is possible, therefore, that in comparing the incidence of developmental coxa vara with that of congenital dislocation of the hip joint our ratio of one to thirteen may be different from that of other clinics.

Familial tendency-This paper is based on fifteen cases admitted to the Children's Hospital, Toronto, and one other case which was first seen at the age of forty-three years. Of these sixteen patients, eight were males and eight were females. In ten, the condition was present on one side; in six, both hips were involved. Of the sixteen patients, four were related: two were brother and sister, and two others were second cousins of these first two. They all had other brothers and sisters, none of whom had any limp. One uncle who was reported to have had a limp could not be traced. None of the other twelve patients were related and none gave any history of a limp in the family.

\section{GLINICAL HISTORY AND PHYSICAL SIGNS OF DEVELOPMENTAL GOXA VARA}

Clinical history-The clinical history was somewhat the same in all cases. In a few, the limp or waddle was noticed when the child first began to walk, but in most it did not appear until the age of three or four years, the gait being apparently normal before that age. The limp was not preceded by any severe injury and it was usually painless. At first it was very slight, but it increased slowly; as a rule it was not until a year later, and usually much more than a year later, that the patients sought advice. In a few cases, long after the limp had developed, there was a history of a sudden attack of moderate pain, coming on after trivial injury, disappearing in a couple of weeks, but leaving definite and lasting increase in the limp. More commonly the limp increased gradually over a period of six or seven years and remained almost painless throughout. In cases which were first seen at a later age, there was a history of some improvement in the limp at the age of about twelve years. One patient, seen at the age of forty-three years with marked bilateral deformity (Fig. 4), had developed

* Paper read at the combined meeting of the American, British, and Canadian Orthopaedic Associations, in Quebec, June 4, 1948.

Vol. $30 \mathrm{~B}$, No. 4 , NOVEMBER 1948 


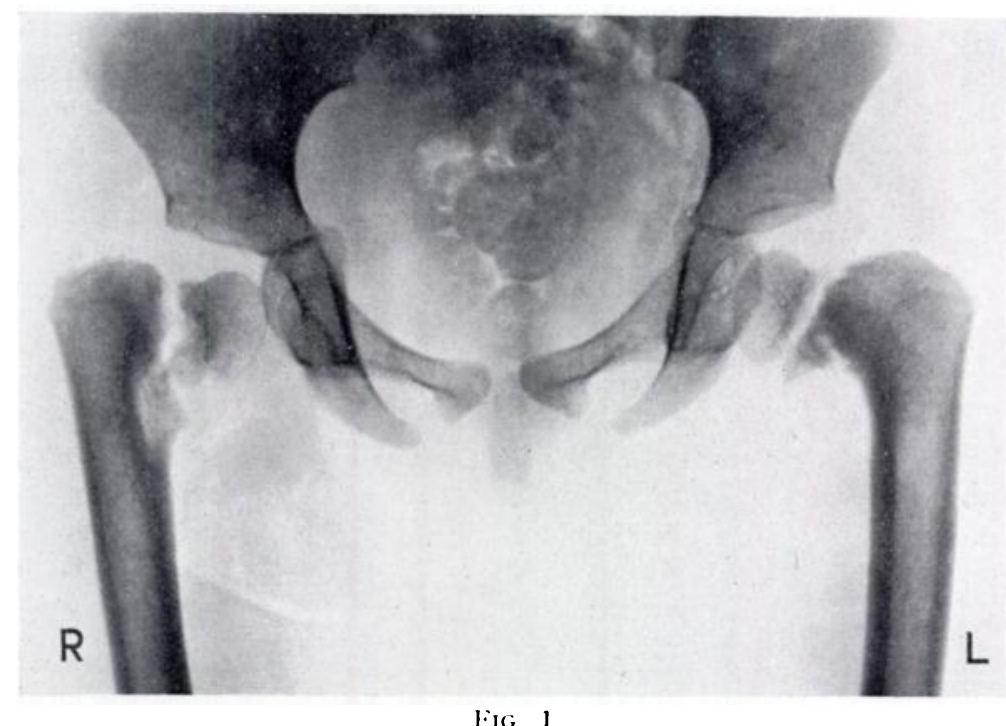

Developmental coxa vara; age four years. On the right, the gap in the bone branches away from the epiphyseal line at its lower end. On the left, it branches into two, leaving a triangular part of bone separated.

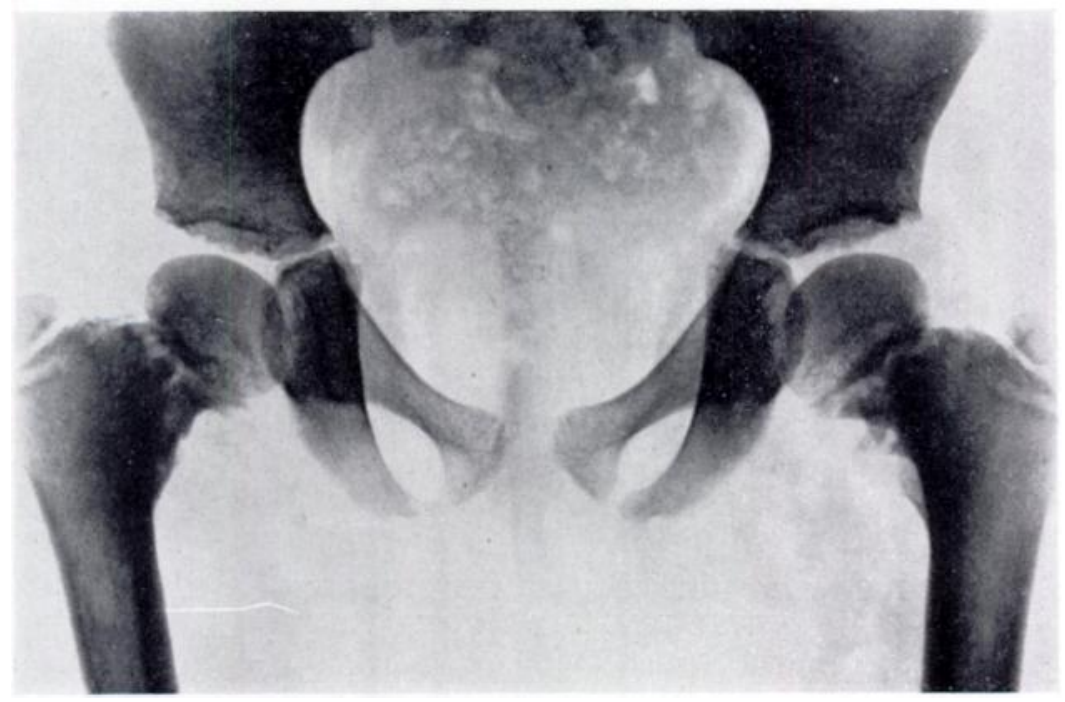

Fig. 2

Bilateral developmental coxa vara first seen at the age of six years, with only slight deformity and with the gap in the bone more horizontal than vertical. The appearance of "fragmentation" is rather marked.

severe pain on one side at the age of forty years, probably because of arthritic changes in the altered hip: the pain was much improved by subtrochanteric osteotomy:

Clinical examination-Physical examination of these patients showed no abnormalities except those which were attributable to the condition of the hips. Most patients were rather short in stature, shorter than other members of the family at the same age, but not to a marked degree. None showed signs of rickets. There was elevation of one or both trochanters with limitation of abduction movement, the degree of limitation depending on the degree of coxa vara deformity. When this deformity was severe, there was also limitation of 


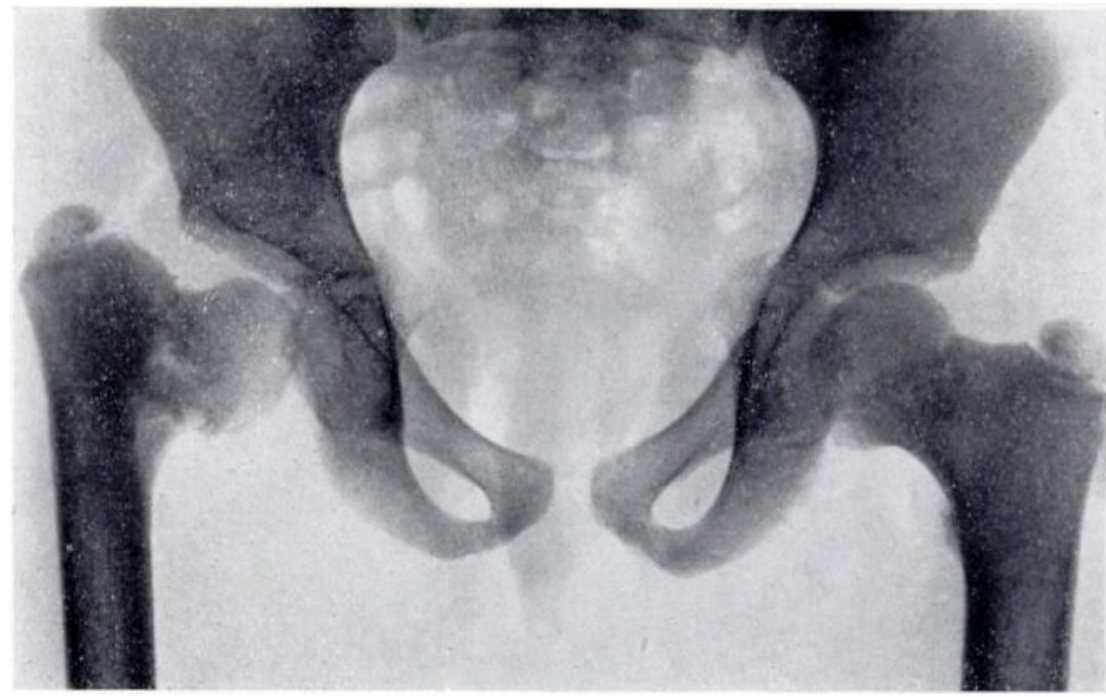

Fig. 3

Developmental coxa vara seen at the age of eight years with rather marked deformity and shortening of neck, particularly at its lower margin, thus giving an impression of collapse of the tissues forming it.
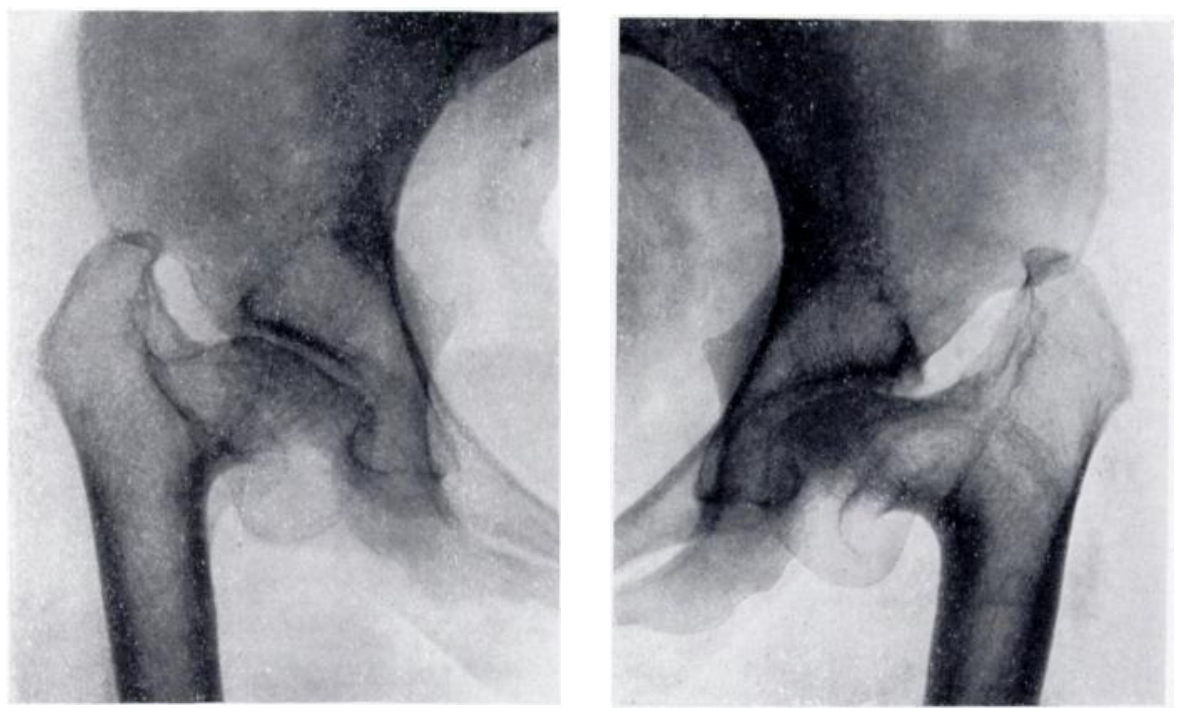

FIG. 4

Developmental coxa vara first seen at the age of forty-three years; no treatment. The gap in the bone has closed, but with marked deformity. The gait was good but there was pain on one side, during the last few years, from secondary arthritis.

extension movement of the hip, and of rotation movement in both directions, but in none was there marked external rotation deformity of the limb such as is seen in slipped epiphysis. In bilateral cases there was moderate lumbar lordosis on standing. The femur below the neck appeared normal and, when only one side was involved, the length of the two limbs as measured from the trochanters was the same.

In early stages the children did not complain of pain, although usually they tired easily. By the time the patient was first seen the limp or waddle was rather marked; it was greater than could be accounted for by deformity alone, and it seemed probable that to some extent 


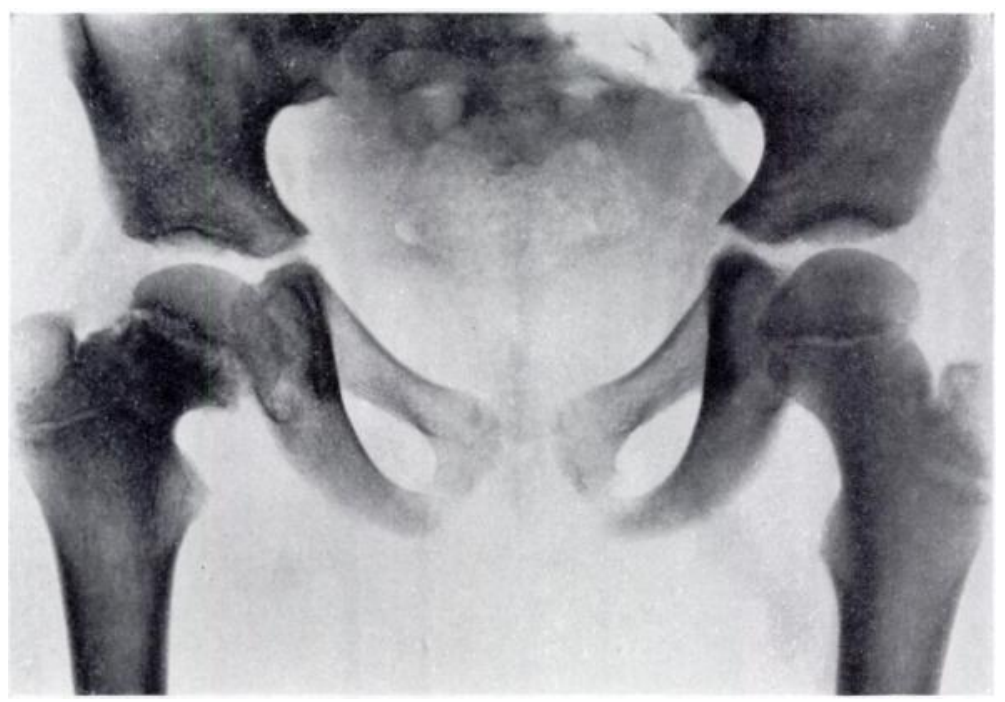

Fili. 5

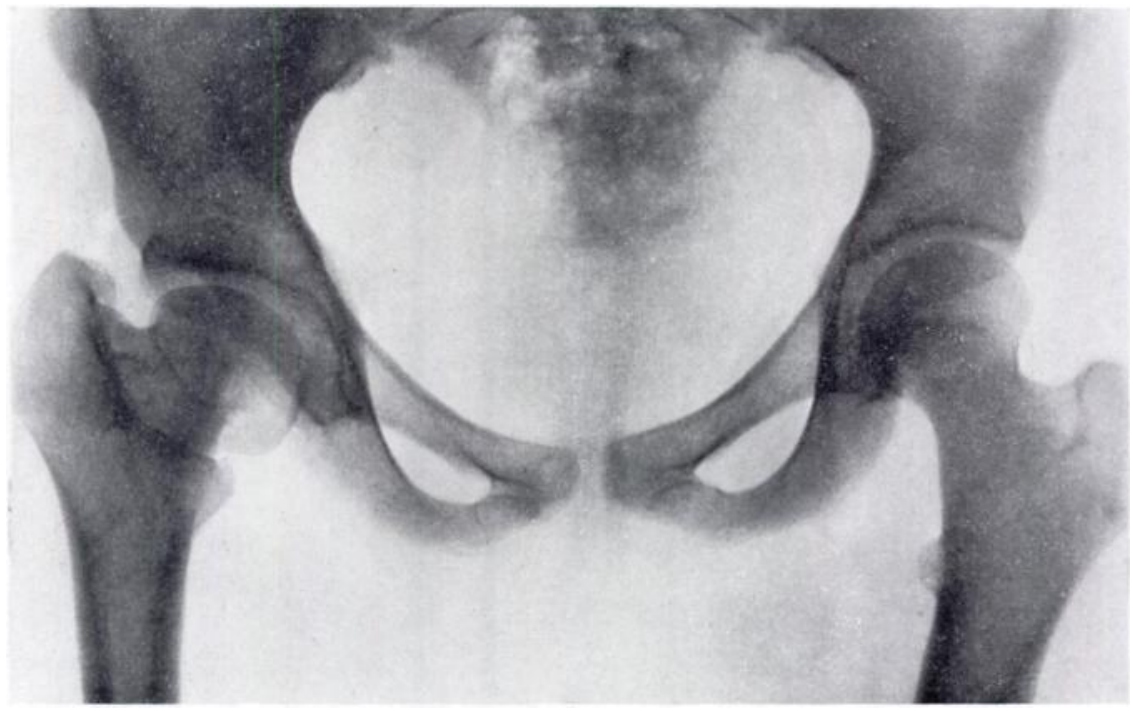

Fig. 6

Developmental coxa vara in a patient who had no treatment. The gap in the bone, shown at the age of twelve years (Fig. 5), closed completely by the age of seventeen years (Fig. 6) with normal looking bone, but with moderate varus deformity and considerable shortening of the neck.

the limp was due to instability of the femoral neck at the gap. In older patients, when the gap in the femoral neck had healed, the gait, having regard to the severe degree of deformity; was extraordinarily good.

Radiographic examination-The radiographic appearances were very similar in nearly all cases. Even in early stages, when the child first began to limp, a gap in the bone was already obvious (Fig. 1). It crossed the neck of the femur distal to the epiphyseal line. In most cases it ran part of its course parallel to the epiphyseal line, but towards one end, usually the lower end, it branched away from it, and in some cases, also usually at the lower 


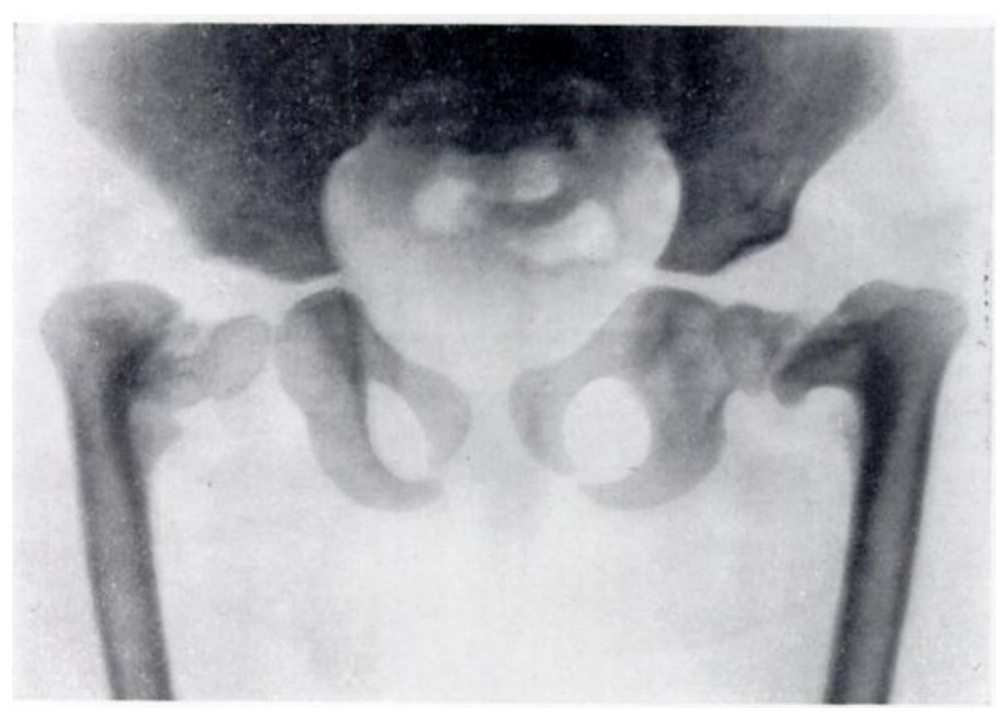

FIG. 7
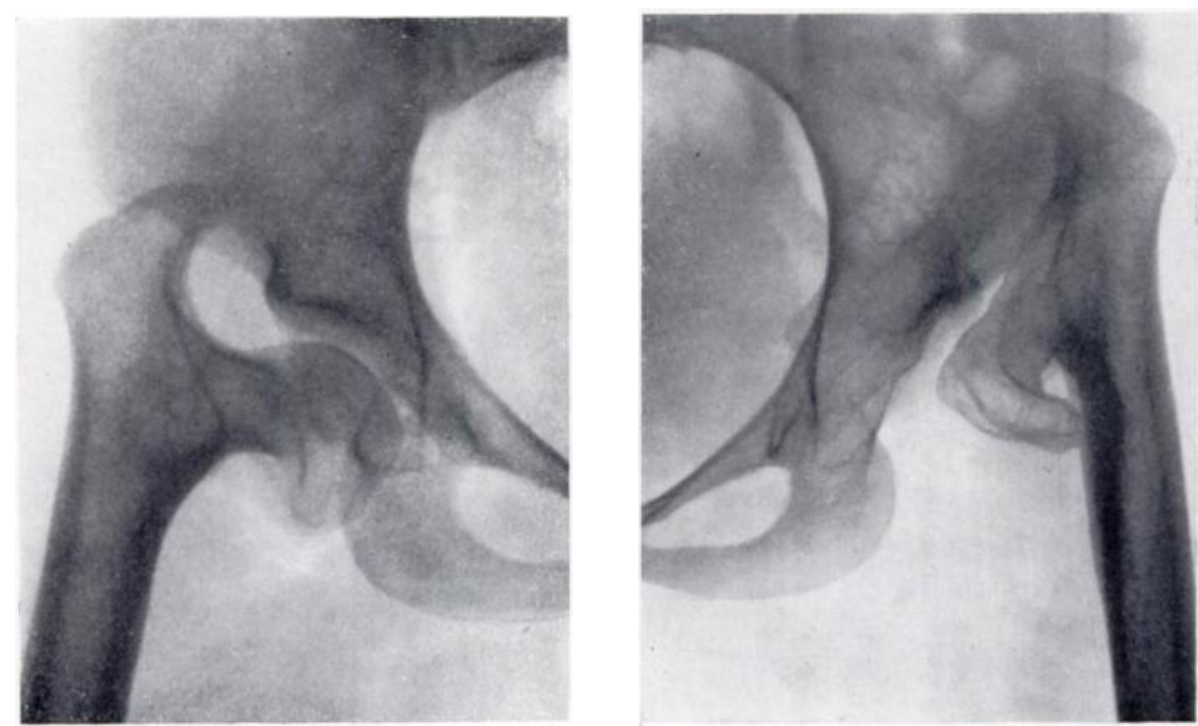

FIG. 8

Bilateral developmental coxa vara at the age of three years (Fig. 7) and at the age of sixteen years (Fig. 8) in a patient who received no treatment. The gap in bone closed completely but with marked deformity. Nevertheless the gait was extraordinarily good.

end, it divided into two, leaving a triangular portion of bone more or less isolated. The gap was not broad and it did not follow a straight line with clear-cut edges; the margins were usually uneven. The bone was abnormal in appearance, particularly just distal to the gap; irregular areas of greater density alternated with areas of lesser density, thus giving rise to the appearance which is often described as " fragmentation." In no case was there increased density of the femoral head suggesting necrosis of the bone such as may occur in slipped epiphysis, or in fractures at this level. As a matter of fact the density of the head was often less than normal. The epiphyseal line was usually narrow, and sometimes it could be seen 


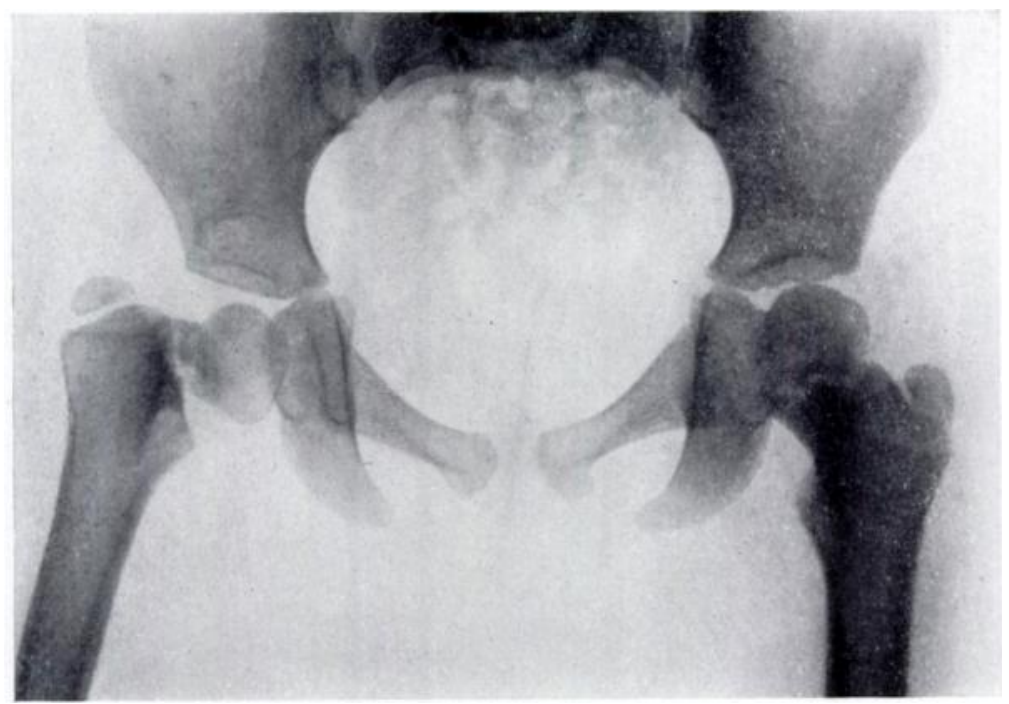

FIG. 9

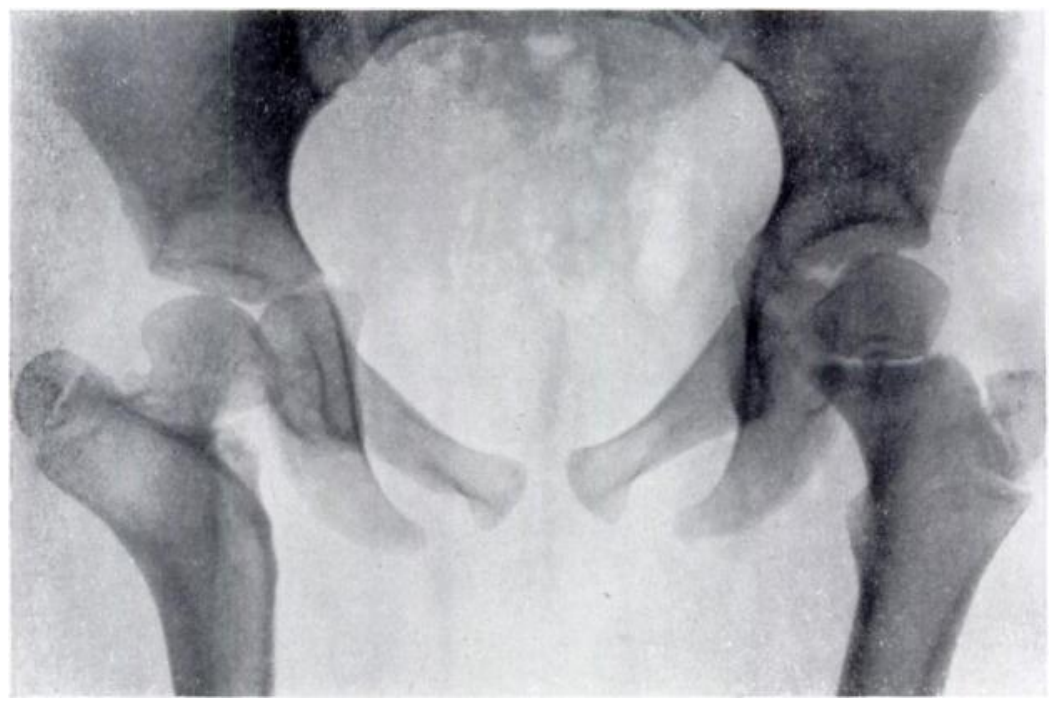

FIG. 10

Developmental coxa vara in a patient, aged six years, before operation (Fig. 9). Same patient two years after subtrochanteric osteotomy (Fig. 10). The gap in the bone has closed completely. There was no limp.

only with difficulty. It should be noted that in this condition the characteristic gap in the bone is not the epiphyseal line: developmental coxa vara must not be confused with slipped upper femoral epiphysis which is more common, and in which deformity occurs at a different level and at a later age.

In some early cases of coxa vara in which the deformity was not marked, the gap in the bone ran in a direction more horizontal than vertical (Fig. 2). As deformity increased the head rotated downwards, and both the gap, and what could be seen of the epiphyseal line, became more vertical. In most cases seen at about the age of eight years varus deformity 


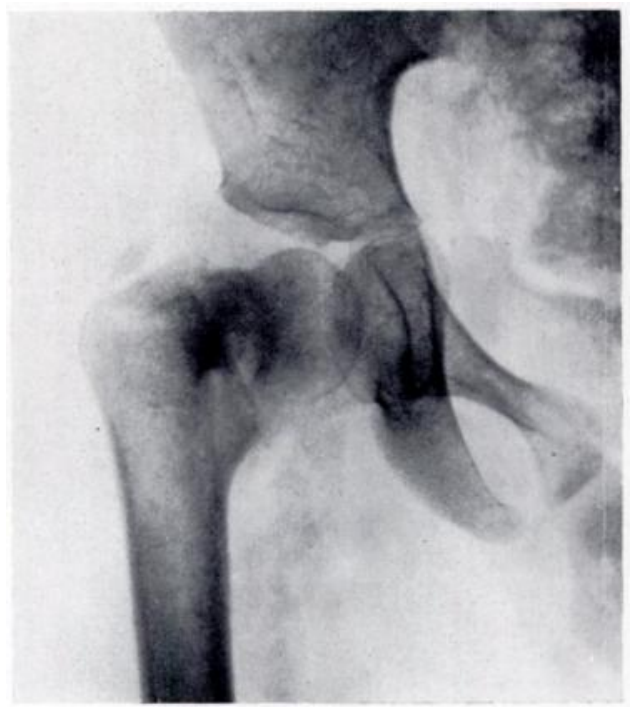

FIG. 11

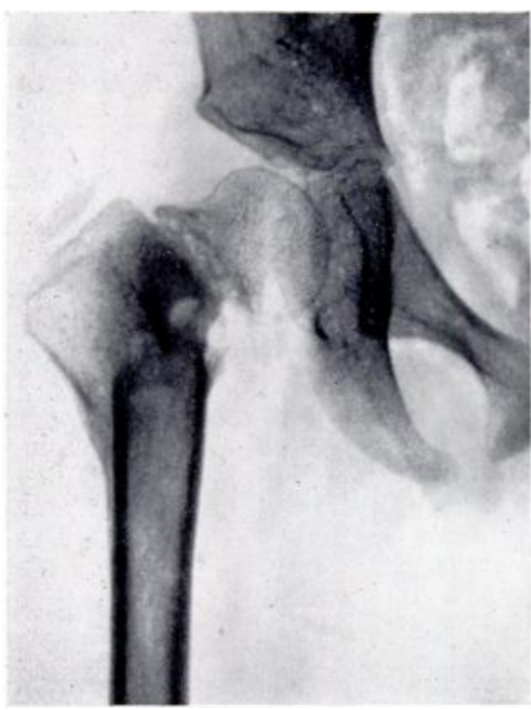

FIG. 12

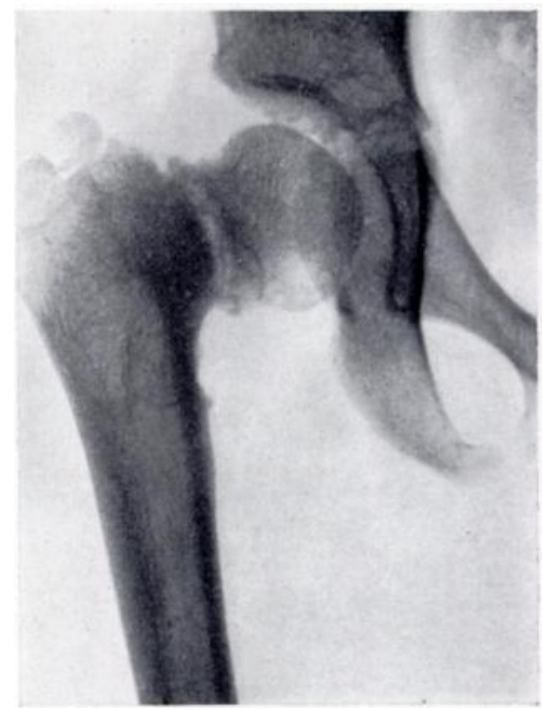

FIG. 13

Coxa vara in patient aged five years (Fig. 11). Fig. 12 shows radiograph two months after osteotomy. Two years later the gap has not closed and the deformity has increased (Fig. 13); probably due to insufficient abduction at the osteotomy.

was rather marked, and the neck was short, particularly at its lower margin, thus giving the impression that there had been collapse of the tissues forming it (Fig. 3). In cases seen at about the age of twelve years the appearance of bone fragmentation was less marked and the gap was narrower. In the three cases which were seen at the age of more than sixteen years the gap had bridged completely and the neck was in one piece with bone of almost normal texture (Figs. 4 to 8). In these untreated cases, the final degree of deformity varied greatly; as a rule it went on to the extreme limit, but occasionally it never progressed beyond the right angle.

vol. $30 \mathrm{~B}$, No. 4 , NOVEMBER 1948 


\section{PATHOLOGY OF DEVELOPMENTAL COXA VARA}

We did not remove tissue for histological examination and we cannot add information as to the origin and cause of this condition. The explanation which is generally accepted is that it is due to faulty development of the neck of the femur, with imperfect formation in cartilage, and with delayed and incomplete ossification of the cartilage. While this explanation does not entirely fit the picture it is probably the correct one. Varus deformity is no doubt caused by gradual bending of the unossified cartilage. Shortening of the femoral neck is due partly to this bending but largely to lack of growth at the epiphyseal line which is never normal in appearance and always undergoes premature fusion.

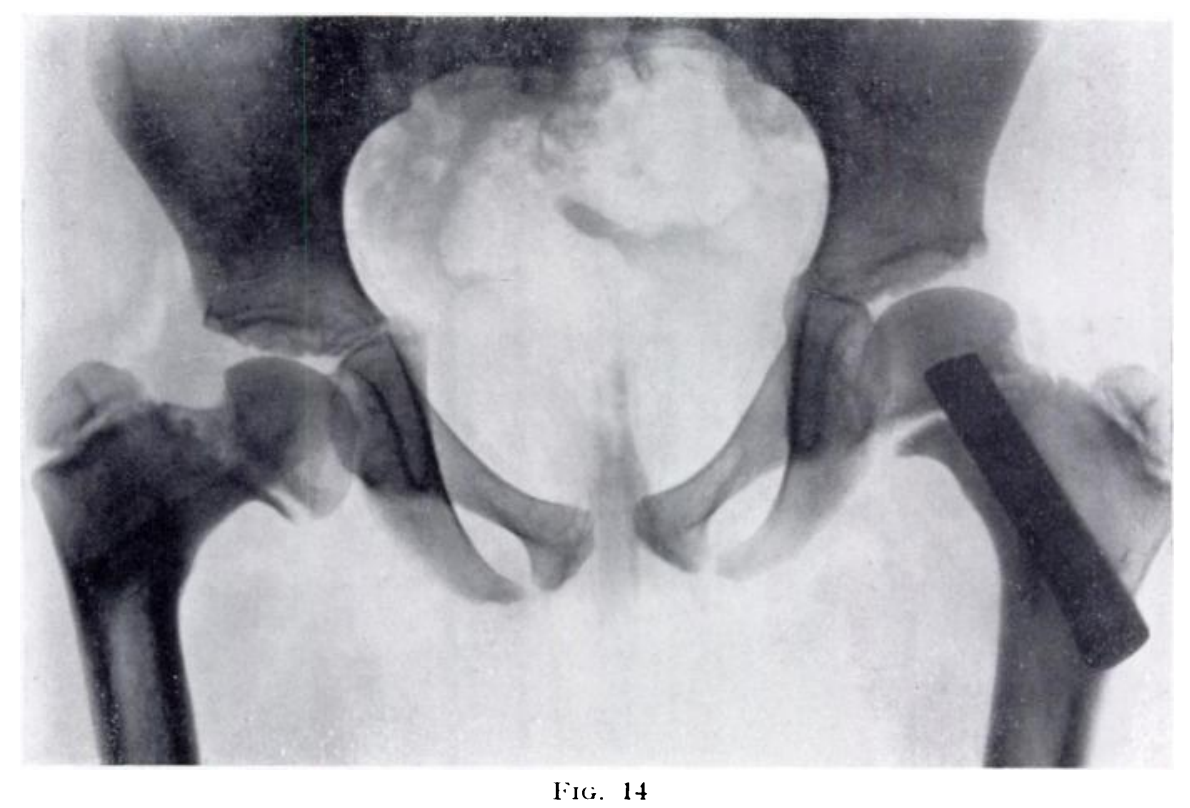

Bilateral coxa vara in a patient aged six years (for original condition see fig. 2) treated by nailing on one side and bone grafting on the other. Radiographs eleven months after operation show considerable closure of the gap on the grafted side, but little closure on the nailed side.

Clinical course-The usual course of the condition is well known. The gap in the bone is present at an early age. Without treatment the deformity increases slowly at a varying rate and $t o$ a varying extent over a period of years. At about the age of twelve years increase in the deformity ceases. Finally, in most cases at any rate, the gap in the bone heals, leaving a deformity which is usually severe but which sometimes is only moderate.

\section{TREATMENT OF DEVELOPMENTAL COXA VARA}

Two factors must be considered in treatment, namely: the coxa vara deformity; and the gap in the femoral neck. The deformity causes shortening of the limb and limitation of abduction movement of the hip. If it is severe it gives rise to a marked limp and should be corrected, or in some way compensated.

Treatment by traction-We have tried to correct the deformity by strong traction and forced abduction at the level where it takes place, that is to say at the gap in the bone. All that we have succeeded in doing is to pull the femoral head almost out of the acetabulum without changing the angle of the neck in the slightest. We have concluded that, although 


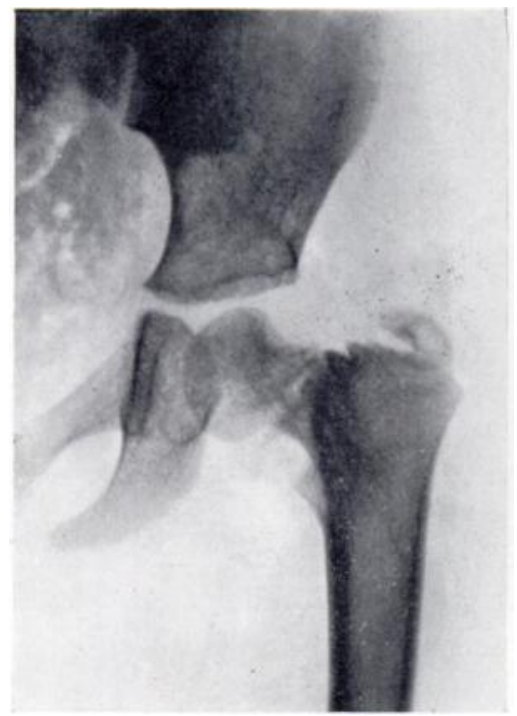

Fisi. 15

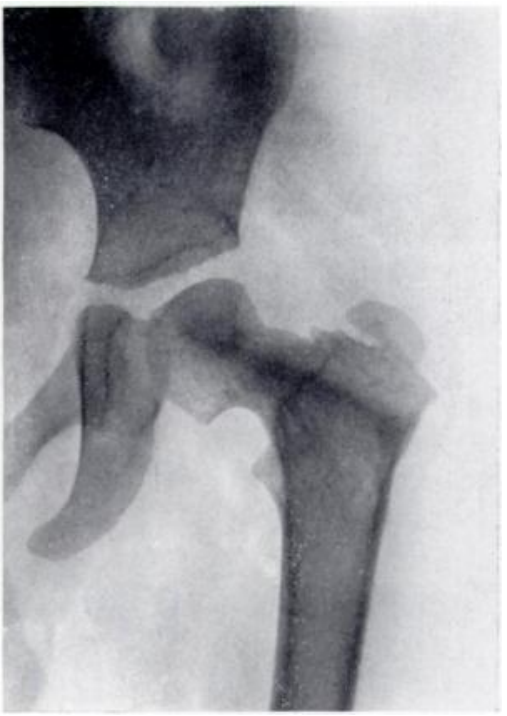

III 816

Developmental coxa vara in patient aged six years (Fig. 15). Three months after bone grafting the gap is almost completely closed (I.ig. 16).

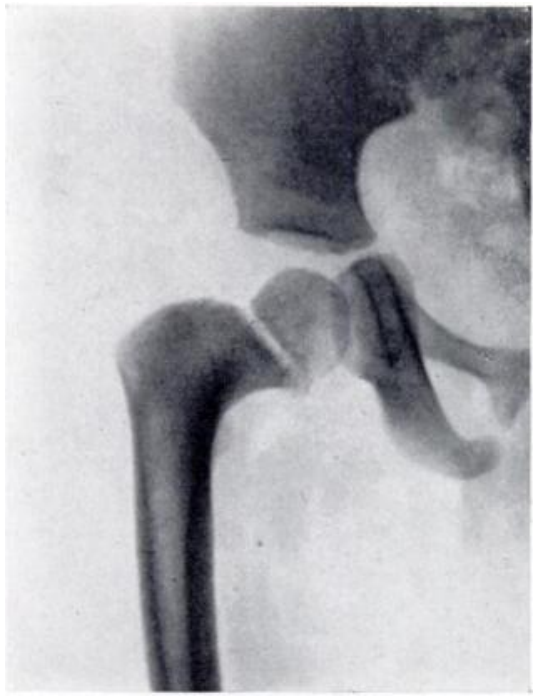

FIG. 17

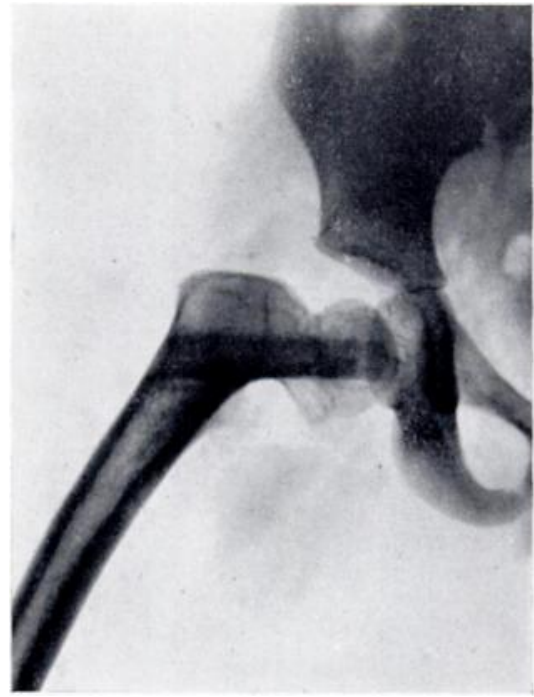

Fig. 18

Coxa vara in a patient aged three years (Fig. 17). Four months after insertion of a bone graft the gap in the bone is closing satisfactorily ( 1 ig. 18). 
deformity may increase gradually, the neck is nearly always too rigid at the gap to be bent straight by closed manipulations. It seems unwise to complete division of the neck at the gap by operation, or to do an osteotomy through the neck distal to the gap; and we have not tried either.

Treatment of late cases by trochanteric osteotomy-Unless the deformity is very severe indeed, limitation of abduction can be overcome and shortening can be reduced by the safer procedure of osteotomy between the trochanters, or below them, the lower fragment being abducted widely. We have performed this operation on twelve hips. The osteotomy always united rapidly and the limp either disappeared or was much improved. Besides overcoming deformity, the abduction osteotomy did something else. In every case that we have been able to follow long enough, with one single exception, the gap in the bone healed completely within a year, and in a much shorter time than would have been possible without operation (Figs. 9 and 10). This healing of the bone defect was probably attributable to change in the line of weight-bearing at the gap from a shearing stress to a compressive force. In the one case that did not heal, it is probable that the fragments were not abducted widely enough at the osteotomy (Figs. 11-13). At the end of two years the gap was still open and the deformity of the neck had increased. Recently another osteotomy has been performed on this patient with more abduction of the fragments.

Importance of wide abduction at the site of osteotomy-The importance of gaining sufficient abduction at the site of osteotomy must be emphasized. This is necessary not only to secure closure of the gap in the femoral neck, but also to overcome the disability caused by deformity. The best results were obtained when the distal fragment was abducted sixty or seventy degrees at the level of osteotomy. In doing the operation, attention must be paid to the relative length of the two limbs. The affected limb is shortened by deformity of the neck. It is, therefore, wise to do the osteotomy between the trochanters, and to prevent shortening due to over-riding of the fragments, either by using some form of internal fixation or by applying traction to the limb after operation. Flexion deformity at the hip joint can be overcome at the time of the osteotomy.

Treatment of early cases by nailing and grafting-Abduction osteotomy is a satisfactory method of treatment for cases in which the coxa vara deformity is bad enough to require correction. It leaves a hip joint which may not be perfect in function, but which is much better than it was, and which is unlikely to get any worse for the reason that the gap in the neck is closed. It must be recognised, however, that some hips are seen before the coxa vara deformity has become at all marked, and before it is bad enough by itself to produce any limp. The diagnosis is made obvious by the radiographic appearance and it is almost certain that, without treatment, deformity will increase steadily until the gap in the bone closes. In these early cases the most important thing is to close the gap before deformity becomes disabling, and it would seem better, if possible, to do this without osteotomy which may distort the mechanics of the hip. Since healing of the gap after osteotomy seemed to be due to elimination of the shearing forces, it was thought that the same result might be obtained by the insertion of a Smith-Petersen nail. This was done in one hip of the patient shown in Fig. 14. After eleven months, the gap was still widely open. In the other hip of the same patient, a few days after nailing the first hip, a large drill was passed up the neck of the femur into the head; into this drill-hole two tibial bone grafts, placed cortex to cortex, were inserted tightly. Eleven months after operation, the gap in the bone was pretty well healed; repair was much more advanced than on the side which had been treated with a Smith-Petersen nail.

Since then two other cases have been treated by grafting; in both the gap is healing well, one after three months, and the other after four months (Figs. 16, 18). It would seem that this is much the best way to secure closure of the gap. The most striking thing about these 
three grafted cases, one with a nail in the other hip, is that all are walking and running normally and all have lost the limp they had before operation, although there has been no change in the degree of coxa vara deformity. It would appear that weakness or instability at the site of the gap in the bone is an important element of the limp, and that if the gap can be closed before the coxa vara becomes sufficiently severe to cause disability, the symptom should disappear. The objection that at this age a bone graft across the epiphyseal line may arrest growth applies very little in these cases, because in any event growth in length here will be almost negligible.

\section{SUMMARY}

Most of what I have said has been said before by various writers. Abduction osteotomy is the recognised form of treatment for developmental coxa vara. The results from this operation are usually good. But the results of treatment would probably be better if the condition could be diagnosed before deformity had become disabling, and if the gap in the bone could be closed by other means than osteotomy. Good results of bone grafting in early cases of developmental coxa vara are reported.

\section{REFERENCES}

BArr, J. S. (1929): Congenital Coxa Vara. Archives of Surgery, 18, 1909.

Dúncan, George A. (1938): Congenital and Developmental Coxa Vara. Surgery, 3, 741.

Elmslie, R. C. (1913): Coxa Vara: Its Pathology and Treatment. London: H Frowde.

Fairbank, H. A. T. (1928): Infantile or Cervical Coxa Vara. Robert Jones Birthday Volume. London: Oxford University Press, 225.

Golding, F. Campbell (1948): Congenital Coxa Vara. Journal of Bone and Joint Surgery, 30-B, 161. Golding, F. Campbell (1938): Congenital Coxa Vara and the Short Femur. Proceedings of the Royal Society of Medicine, 32, 641 .

Noble, Thomas P., and Hauser, Emil D. (1926): Coxa Vara. Archives of Surgery, 12, 501.

Ollerenshaw, Robert (1938): The Femoral Neck in Childhood. Proceedings of the Royal Society of Medicine, 32, 113.

ZADEK, IsAdORE (1935): Congenital Coxa Vara. Archives of Surgery, 30, 62. 